Original Research Paper

\title{
An Effect of the World Health Organization's "My Five Moments for Hand Hygiene": An Initiative on Hand-Hygiene Knowledge, Beliefs, Attitudes and Compliance Among Certified Nursing Assistants Working in A Health Care Facility
}

\author{
Catherine Turkett Kamara, DrPH \\ Public Health, CE Turkett Consultants, LLC, USA
}

\author{
Article history \\ Received: $15-04-2020$ \\ Revised: 27-04-2020 \\ Accepted: 04-05-2020
}

Email: info@saltrehabcliniclib.org

\begin{abstract}
According to the $\mathrm{CDC}$, an estimated four million Americans reside in long-term care facilities in the United States, among which one to three million life-threatening infections occur every year. These infections at long-term care facilities are a major cause of hospitalizations and death. The purpose of this research project was to provide a nonrandomized pretest/posttest on knowledge, belief, attitudes and compliance to all 45 CNAs selected for this study to improve current hand hygiene practices of certified nursing assistants employed by a health care facility. Pre-test and posttest assessments were in addition to an intervention approach administered after the pretest and before the posttest. The World Health Organization Hand Hygiene Knowledge Questionnaire for Health Care Workers, the Hand Washing Self-Assessment Inventory Scale Questionnaire for belief and attitude and a wall hand sanitizer as a proxy to measure hand compliance were the instruments. The intervention administered was the WHO's "My Five Moments for Hand Hygiene" approach to improve hand hygiene practice and compliance. The participants included $30 \mathrm{CNAs}$ selected as the intervention group and 15 $\mathrm{CNAs}$ as the control group. The results indicated that the mean difference $0.14,95 \%$ CI $[0.05-0.23)$ in Knowledge between the treatment and control groups was significant $(p=0.004)$. The results of the 2 by 2 factorial ANOVA conducted to determine Belief and Attitude indicated a significant main effect $\left.\left(F_{1,43}\right)=30.55, p<0.001, p \eta^{2}=0.42\right)$. The analysis for Attitude indicated a significant main effect $\left.\left(F_{1,43}\right)=29.37, p<0.001,{ }_{p} \eta^{2}=0.42\right)$. For the hand sanitizer measurement, there was no significant difference in hand sanitizer use between control and intervention floors at baseline $(p=0.175)$. After the intervention training, the intervention floors had significantly more hand sanitizer use than the control floors $(p=0.033)$.
\end{abstract}

Keywords: Accountability, Buddy System, Certified Nurse Assistants, Compliance, Hand Hygiene, Infection, Microorganism, Morbidity, Mortality

\section{Introduction}

The CDCP (2016) estimated that four million Americans reside in health care facilities. The CDC's data are limited but estimates in the medical literature indicate that one to three million life-threatening infections occur in health care facilities every year. These infections are a major cause of hospitalizations and death; about 380,000 health care facilities residents die from infections each year. A study by Pulliam (2015) revealed that a facility in the South Atlantic United States reported 185 cases of HAIs over a 12-month period. However, after the use of photocatalytic coating intervention, the infection rate decreased by $30 \%(55.5, p$ $=0.02$ ). According to NHHV (2018), the Department of Community Health, in the South Atlantic United States, that oversees nursing homes in the state reported that federal inspection records showed that 
over $40 \%$ of its nursing homes had been cited for infection-related issues.

This research project was an attempt to improve hand hygiene practice and compliance among Certified Nursing Assistants (CNA) in a health care facility; to introduce a hand hygiene intervention and expand on previous studies, as a way to reduce infection rate. This was accomplished by using the World Health Organization (WHO) "My Five Moments for Hand Hygiene" approach as an intervention. This approach is endorsed by the Centers for Disease Control and prevention (CDC) as a best-practice training for hand hygiene that results in immediate improvements in compliance (Moghnieh et al., 2017). This research project also provided a nonrandomized pretest and posttest assessment of 45 CNAs for their knowledge, beliefs, attitudes and compliance regarding hand hygiene. The assessments involved administering the WHO Hand Hygiene Knowledge Questionnaire for Health Care Workers and the Hand Washing Self-Assessment Inventory Scale Questionnaire and treated wall hand sanitizer use as a proxy for compliance. This research employed the Theory of Planned Behavior (TPB) as the theoretical framework. Ajzen and Fishbein (1980). Ajzen (1985) then developed the theory. According to the TPB, beliefs, behaviors, attitudes, subjective norms, normative beliefs and perceived behavior control are all constructs that together shape an individual's behavioral intentions and behavior (O' Boyle et al., 2001). In addition, there are assumptions of the instruments that were used in this research. These include the assumptions that the WHO Hand Hygiene Knowledge Questionnaire for Health Care Workers would measure change in knowledge in the pretest and posttest; that the Hand Washing SelfAssessment Inventory Scale Questionnaire in a pretest and posttest would measure personal self-assessment to hand hygiene compliance; and that the use of wall hand sanitizer as a proxy in a pre- and post-check would serve as a good surrogate for measuring hand hygiene compliance. This research project had limitations as well. For example, the sample size in this study is small because the 45 CNAs were selected from a single health care facility site. Therefore, the results of this study are limited to the sample only and not the entire population of CNAs.

NHHV (2018) also noted that the Federal Center for Medicare and Medicaid Services requires health care facilities to have systems in place to prevent infection. According to Article 483.65 "the facility must establish and maintain an infection control program designed to provide a safe sanitary and comfortable environment to help prevent the development and transmission of disease and infection". (p: 51). Therefore, a simple and reliable monitoring system for hand hygiene compliance at this facility is critical. The apparent lack of an effective compliance monitoring system indicated a need to put a reliable plan, such as the "My Five Moments for Hand Hygiene" intervention, into place.

\section{Background of the Study}

Hand hygiene is well-established as an effective measure to prevent health care-associated infections (Gould et al., 2017). When using the "My Five Moments for Hand Hygiene" intervention, it is important to present hand hygiene practices in a simple manner for maximum compliance. Moghnieh et al. (2017) found that this intervention resulted in a significant increase in compliance for the incentive-driven group: $60 \%$ to $77 \%$ at its peak in Week 14 compared to $21 \%$ for the baseline group. After the intervention ended, compliance dropped by $34 \%$ by Week 21 compared to $43 \%$ in Week eight $(p<0.0001)$.

Al-Khawaldeh et al. (2015) investigated nursing students' hand hygiene compliance. They concluded that "knowledge of, belief about and attitude towards Hand Washing were the significant variables predicting compliance with Hand Washing" (Al-Khawaldeh et al., 2015; p: 572). The WHO has continued to stress the importance of hand hygiene and made its hand hygiene initiative a core element of patient safety in its 2005 patient safety challenge "Clean Care is Safer Care" (WHO, 2017), which was aimed at expanding a national hand hygiene campaign worldwide. Shinde and Mohite (2014) reported that although hand hygiene is the primary measure for preventing cross-transmission of microorganisms, only $40 \%$ of health care workers at a tertiary-care hospital complied with hand hygiene regulations.

The "My Five Moments for Hand Hygiene" intervention was introduced through an interactive presentation to train participants on their responsibilities and on how to increase hand hygiene compliance through peer accountability. The intervention focused on establishing a collaborative effort through a buddy system for CNAs to promote compliance with hand hygiene practices. The "My Five Moments for Hand Hygiene" approach was also introduced as a very functional hand hygiene compliance tool because it can be easily used to determine if hand hygiene compliance is being practiced correctly among different groups of health care workers (Melo de Souza et al., 2015). The effectiveness of the "My Five Moments for Hand Hygiene" intervention could also be monitored. In one study using the "My Five Moments for Hand Hygiene" approach at a tertiary hospital in Shiraz, Iran, results of the study indicated a significant change in hand hygiene compliance: $70.98 \%$ after the intervention was introduced compared to only $29.8 \%$ before the intervention was introduced (Farhoudi et al., 2016). The WHO First Global Patient Safety Challenge "Clean Care is Safer Care" was initially developed to adapt the Swiss national hand hygiene campaign for worldwide use (Sax et al., 2007). Today, the "My Five Moments for Hand Hygiene" approach has expanded to many countries in the world. In a hospital in Nigeria, West Africa, where the "My Five Moments for 
Hand Hygiene" intervention had been introduced, healthcare workers were observed for compliance in multiple settings: Surgery ("before patient contact," WHO, 2007), anesthesiology, Intensive Care Units (ICUs) and among senior nurses (Shobowale et al., 2016).

\section{Research Design}

The study was designed as a quasi-experiment (purposive sampling) research project using a quantitative pretest and posttest control approach to study and administered the evidenced based "My Five Moments for Hand Hygiene" training to improve hand hygiene compliance. Thirty rooms were monitored in a pre- and post-check for wall sanitizer usage as a proxy for hand hygiene compliance. Forty-five Certified Nurses' Assistants were selected by the education specialist at this facility and participated in the pretest and posttest using the WHO Hand Hygiene Knowledge Questionnaire and the Hand Washing Self-Assessment Inventory Scale Questionnaire. Thirty CNAs formed the intervention group and participated in the intervention training after the pretest and before the posttest. Fifteen CNAs formed the control group and participated in the pretest and posttest but did not take part in the intervention training. A brief introduction of the study, including its methodology and purpose, was provided and explained to the participants. Both males and females participated in the study and participants' ages ranged from 24 to 57 years old. The racial and ethnic background was diverse and included African American/Black, Filipino, South Indian and Caucasian participants.

Inclusion criteria were those CNAs in nonadministrative roles who were purposely selected for this study. The rationale for the selection process was to discourage any personal biases from participants serving in administrative roles in order to prevent a label of conflict of interest. The employees selected during this process were expected to have no apparent conflict of interest and to participate fully in this study without any biases.

An exclusion criterion for this study included but was not limited to other direct patient care providers like physicians, other nursing staff and allied health workers at this health care facility, as well as the lack of technology device use as part of the intervention.

\section{Impact and Attitude Toward Reducing Infection in Hospital Settings}

In 2016, the CDC cited there were 721,800 HAIs cases. Such infections occur and spread through unclean hospital settings and infected hospital equipment, as well as lack of adherence to anti-infection strategies, such as good hygiene for hands (CDCP, 2016). HAIs have received extensive attention from hospitals and remain a crucial issue for patient care and a preventable high cost. In 2010, the CDC and Medicaid and Medicare services set a target to reduce the number of HAIs by $40 \%$ in 2013 , hoping to reduce patient injuries by 1.8 million (CDCP, 2016). Due to such an important issue of patient care, in October 2014, health care institutions with the highest percentage of infection transmitted by the hospital were fined $1 \%$, in addition to other federal fines. Health care institutions can invest in educating medical staff to prevent infections, apply tried and tested strategies for health-related infections such as hand hygiene and develop strategies to improve compliance with infections.

However, HAI prevention is more dependent on the culture of hospitals, leadership and organizational involvement in preventing infections on procedures and programs (HAI, 2014). It was found in research that the horrendous HAIs in a hospital in the USA required changing the cultures of medical personnel by carrying out a qualitative study (CHC, 2010). According to the article, Dr. Pronovost, professor of anesthesiology and critical Medicine at the John Hopkins University medical School, believes that the control lists and guidelines for preventing infection are ineffective because they are not applied consistently.

Moreover, the research (CHC, 2010; p: 14) suggested that most doctors and nurses agree to apply checklists and guidelines for preventing infections but sometimes nurses and or doctors forget to apply them. However, if a doctor forgets and a nurse is asked to speak and indicate that the doctor must comply, the nurse(s) becomes reluctant to correct doctors. Some of the answers from the nurses were "it is not my job to police doctors and if I do, they will take my head off" (CHC, 2010; p: 14). Doctors would answer, "You can't have a nurse secondguessing me in public, it seems that I don't know anything" (CHC, 2010; p: 14). In order to change poor compliance attitude to reduce infection rate and improve communication and teamwork between nurses and doctors, a program has been developed, which includes both nurses and doctors, with a strong emphasis on training health care providers on how best and informed decisions can be made to protect patients from infection (CHC, 2010; p: 14). The program developed from this research regarding reducing the rate of infection through checklist compliance, encourages the opinions of doctors, nurses, administrators, pharmacists, social workers and security officers of patients. In general, the program promoted ownership spread the responsibility of protecting patients from infections to all hospital occupations. With the new program, all patient safety problems are discussed and addressed as a team (CHC, 2010). Dr. Pronovost understood the need to change the culture of widespread arrogance in the health care sector and thus founded a program at his health care facility to 
reduce infections by altering the culture of open communication between nurses and doctors.

At the beginning of the program, infection rates for central-linked infections were around 19 per 1,000 days of catheters, which were classified as one of the worst in the country (CHC, 2010). However, with the change in culture of teamwork and communication improvement, the hospital has virtually eliminated catheters infection up to 7-per-1, 000 catheters (CHC, 2010). Further studies on the culture of health care institutions and their relationship with hospital achievements have shown that the relationship between nurses and doctors could be the result of infection related to health care (Boev and Xia, 2015).

Boev and Xia (2015) aimed to create an analysis of the relationship of compliance, communication and teamwork between nurses and doctors 'collaboration to reduce the rate of infections acquired in patients. In the hospital setting, these methods were secondary analyses, which consisted of 5-year-old nurse attitudes and approach data from 671 studies on four ICUs, analyzing fans of ventilator-related pneumonia and catheter related middle blood. A multi-level research project was used to assess the relationship between nurses and doctors 'collaboration with HAIs. For every 0.5 units in the nursing and medical association, the percentage of blood infections decreased by $2.98(P=0.005)$ and pneumonia by $1.13(P=0.005$; Boev and Xia, 2015). Overall, the study concluded that the collaboration between nurses and physicians, with regards to the hospital guidelines checklist compliance, would significantly impact compliance and reduce the infection rate of patients in the hospital.

\section{Impact of Hand Hygiene on the Reduction of Hospital-Acquired Infections}

An evidence-based research study conducted in a teaching hospital in Taiwan demonstrated that the implementation of Hand Hygiene Programs (HHPs) reduces preventable HAIs (Chen et al., 2016). This study implemented a 4-year hospital-wide hand hygiene program in Taiwan-based hospitals with high HAI rates. This program stressed the use of alcohol-based hand rubs in Taiwan hospitals and compliance was determined by direct observation and the use of alcohol-based rub products.

A Poisson regression investigation was initiated to analyze the consistency and trends of HAIs during the research study pre-intervention period (January 1999March 2004) and intervention period (April 2004December 2007). According to Chen et al. (2016), 8,420 opportunities for hand hygiene were observed during the course of the research study. Overall, compliance levels improved from $43.3 \%$ in 2004 to $95.6 \%$ in 2007 and were fundamentally associated with increased usage of the alcohol-based hand rubs during the intervention period. Hospitals in Taiwan experienced an $8.9 \%$ reduction in HAIs and the development of MRSA and other multidrug-resistant organisms. The conclusion drawn from this research study was that hand hygiene programs decreased preventable HAIs and challenges while increasing compliance with hand hygiene.

Other research studies completed with regards to examining and analyzing the impact of hand hygiene on decreasing HAIs have revealed that hand hygiene compliance had been linked to the decrease of HAIs (Gould et al., 2017). A research study conducted by Monistrol et al. (2012) to examine the impact of hand hygiene compliance with the use of alcohol-based hand rub revealed a lower incidence of new HAIs after a 12month sustained increase in hand hygiene compliance in three internal medicine wards. During the research study, hand hygiene practices of nurses and physicians were monitored during routine patient care using the WHO's 5 Moments for Hand Hygiene model. Alcohol-based hand rub use was monitored and recorded, and all HAI risk factors were subsequently documented, and occurrence density was found (Monistrol et al., 2012).

The study consisted of 1,693 patients, in which 1,531 opportunities for hand hygiene was observed during the 12-month duration of the research project. The study revealed that hand hygiene improved from $54.3 \%$ to $75.8 \%$ ( $p=0.005)$, also alcohol-based hand rub usage increased from $10.5 \%$ to $27.2 \%$. Overall, the research study concluded that constant hand hygiene and the uninterrupted increase in usage of alcohol- based rubs decrease the frequency of new HAIs (Monistrol et al., 2012). A study conducted by Fox et al. (2015) examined a new hand hygiene protocol designed to decrease hospital infection rates revealed that improvement in nurses' hand washing compliance reduced infections during a 12-month period. This was a pre- experimental research study that intended to analyze and compare 12month rates of two specific HAIs-catheter-associated urinary tract infection and central catheter-associated bloodstream infection-as well as nurses' hand hygiene compliance before and during the use of this new hand hygiene protocol. Research studies on the impact of hand hygiene as an effective strategy in decreasing HAIs have proven that the prevention of HAIs requires consistent hand hygiene compliance and overall quality improvement efforts to effectively phase out these infections (Fox et al., 2015). In a study of evidence based research, a Taiwanese educational hospital showed that the implementation of Hand Hygiene Programs (HHPs) reduces preventable HAIs (Chen et al., 2016). The study carried out a 4 - year hand hygiene program in Taiwan-based hospitals, which had high HAI rates. Moreover, the program highlighted the use of rubbing alcohol in Taiwan's hospitals and direct monitoring and using alcohol-based rubbing products determined 
compliance in order to analyze the coherence and tendencies of the HAIs during the pre-interventional studies (January 1999-March 2004) and the phase of intervention (April 2004-December 2007). Overall, the level of conformity increased from $43 \%$ in 2004 to $95.6 \%$, in 2007. Thereby, increasing the level of conformity with the use of alcohol rubbing during the period of intervention.

Taiwan's hospitals have seen an $8.9 \%$ decrease in HAIs and the development of Methicillin-Resistant Staphylococcus Aureus (MRSA) and other multi-unit organisms. According to the study, manual hygiene programs reduce preemptive HAIs and challenge them, while at the same time increasing adherence to the hygiene of the hands. Other studies that have been completed on the study of and analyzed the effects of hand hygiene on the decline of HAIs have shown that the conformity of hand hygiene has been associated with the collapse of HAIs (Monistrol et al., 2012).

\section{Power Analysis}

The required sample size for the study was determined by a power analysis conducted using the $\mathrm{G}^{*}$ Power software. The sample size was calculated based on Cohen's effect size, the level of significance (alpha level) and the power of the study, which is the likelihood of rejecting a false null hypothesis. A power of 0.80 is normally used in quantitative research to provide valid statistical results (Faul et al., 2009). A medium effect size was used in the power analysis so that it would be neither too strict, nor too lenient. A prior power analysis was conducted considering the statistical test of the 2 by 2 factorial ANOVA using the following means: Difference between two dependent means (matched pairs), a statistical power of 0.8 , a medium effect size of 0.5 for a 2 by 2 factorial ANOVA and a level of significance or alpha threshold of significance of 0.05 .

This yielded a minimum sample size of 45 . Sample size was derived by identifying the most long-term floors, with active rooms in the facility to monitor the wall-sanitizer usage. For participants in this study, 45 CNAs were selected from different floors during the most critical shift, when point of services is performed the most. Note that only 30 CNAs actually participated in the intervention, to test the hypothesis that the intervention would lead them to do better on the posttest than those who did not participate in the intervention or perhaps not; or the hypothesis suggested that the control group 15 CNAs would also improve in the posttest, even though they were exempt from the intervention or perhaps not. The hypothesis also suggested that changes would be detected in wallsanitizer use in 30 rooms on different floors after the intervention or perhaps not Table 1 .
Table 1: G*Power a priori: Compute required sample size for repeated measures, between factors ANOVA

\begin{tabular}{lr}
\hline Parameter & Value \\
\hline Input: & \\
Effect size f & 0.50 \\
$\alpha$ err prob & 0.05 \\
Power (1- $\beta$ errprob) & 0.80 \\
Number of groups & 2.00 \\
Number of measurements & 2.00 \\
G*Power ANOVA “continued" & \\
Corr among rep measures & 0.50 \\
Output: & \\
Noncentrality parameter $\lambda$ & 8.17 \\
Critical F & 4.26 \\
Numerator df & 1.00 \\
Denominator df & 24.00 \\
Total sample size & 26.00 \\
Actual power & 0.81 \\
\hline
\end{tabular}

\section{Data Collection}

\section{Collection Procedure to Measure Compliance}

To measure compliance, current wall handsanitizer usage was investigated on three floors. Different floors of the facility were treated as a proxy for hand-hygiene compliance. A total of thirty wall sanitizers (425 g or $15 \mathrm{oz}$ each) in 10 rooms on each floor were monitored for 14 days in a pre-check before the intervention and 14 days in a post check after the intervention to see whether sanitizer usage increased after the intervention.

\section{Collection Procedure to Measure Hand Hygiene Knowledge, Compliance, Belief and Attitude Using Questionnaires}

A pretest was given using the WHO Hand Hygiene Knowledge Questionnaire for Healthcare Workers. Of this instrument's 21 questions, 10 multiple-choice yes-or-no questions (nos. 12-21) were about hand hygiene; each participants' total score on these 10 items was obtained to measure hand-hygiene knowledge. The other 11 questions were related to participant demographics. The results were evaluated using the SPSS software. A score of $75 \%$ or higher was considered good ("excellent knowledge"), 50\% to $74 \%$ less good (not sufficient knowledge) or "moderate knowledge" and less than $50 \%$ poor (not adequate knowledge).

The content validity of this questionnaire was determined by a review panel of experts (Nabavi et al., 2013). Face validity was established from a pilot test of medical students and reliability was determined to be 0.77 (Nabavi et al., 2013). Indices of the scales were assessed using Cronbach's alpha.

A pretest using the Hand Washing Self-Assessment Inventory Scale Questionnaire was also conducted. This 
asked participants to estimate the percentage of occasions on which they personally complied with hand-hygiene standards in nine specific circumstances. This questionnaire was also assessed using the SPSS software. Participants' hand-hygiene performance was estimated as a percentage.

A posttest was also conducted with the WHO questionnaire to determine whether hand-hygiene knowledge had changed after the intervention in either the intervention or control group. Another posttest was conducted with the hand washing self-assessment to determine whether personal estimates of hand-hygiene performance had changed in either group.

\section{Data Analysis}

This study used a $2 \times 2$ repeated measures (before and after training) with grouping factor (control, treatment) ANOVA. After data collection and entry, the statistical analysis was performed using SPSS (v26). The data were screened for outliers and meeting the assumptions of the two-way factorial ANOVA. The data were found to meet the assumptions and there were no outliers.

The investigation of the mean difference was carried out after significance was observed to determine that the intervention was in fact effective in improving hand-hygiene knowledge, self-assessed hand-hygiene performance and compliance as measured by wall-sanitizer usage.

The means of the posttest should be significantly greater than those of the pretest to show that an intervention was effective. The $p$-value of the Levine's test should be greater than 0.05 to show homogeneity of variances.

The World Health Organization hand hygiene knowledge questionnaire resulted in a time by group interaction $\left(F_{1,43}=4.27, p=0.045, p \eta^{2}=0.09\right)$. The analysis of simple main effects found that mean difference $0.14,95 \%$ CI $[0.045-0.23)$ between the treatment and control groups was significant $(p=0.004)$. Such that the intervention group had a higher mean baseline score as shown in Table 2 . The mean difference $0.042,95 \%$ CI [0.02-0.07) was also significant, where the Intervention group continued to have a higher mean score than the control group. Continuing the analysis of simple effects, the control group had a significant increase in the mean hygiene score (mean difference $=$ 0.25 , 95\% CI [0.18-0.33); $p<0.001)$. The intervention group also showed a significant increase in the mean difference of the hygiene score $0.15,95 \%$ CI [0.1 - 0.21); $p<0.001$ ). In examining the results as presented in Table 2 the intervention group scored higher than the control group at baseline and improved their hygiene scores with the intervention. On the other hand, the control group while having lower hygiene scores had a larger increase in hygiene scores in the post treatment evaluation.

\section{Belief}

Analysis of the beliefs found a significant main effect $\left(F_{1,43}=30.55, p<0.001,{ }_{p} \eta^{2}=0.42\right)$. The main effect for group was not significant $\left(F_{1,43}=1.86, p=0.18\right),{ }_{p} \eta^{2}=$ $0.04)$, nor was there as significant interaction $\left(F_{1,43}=\right.$ $\left.0.74, p=0.4,{ }_{p} \eta^{2}=0.02\right)$. Both groups had similar belief scores that increased between baseline and post treatment testing (Table 2).

\section{Attitude}

Analysis of the Attitude found a significant main effect $\left(F_{1,43}=29.37, p<0.001,{ }_{p} \eta^{2}=0.42\right)$. The main effect for group was not significant $\left(F_{1,43}=2.38, p=\right.$ $\left.0.13,{ }_{p} \eta^{2}=0.05\right)$, nor was there as significant interaction $\left(F_{1,43}=0.87, p=0.4,{ }_{p} \eta^{2}=0.02\right)$. As shown in Table 2, both groups had similar belief scores that increased between baseline and post treatment testing.

Table 2: Means and standard deviations of the hand washing measures comparing the control and treatment groups before and after the training period

\begin{tabular}{|c|c|c|c|c|c|c|c|c|}
\hline \multirow[b]{3}{*}{ Variable } & \multicolumn{4}{|c|}{ Control $(n=15)$} & \multicolumn{4}{|c|}{ Treatment $(n=30)$} \\
\hline & \multicolumn{2}{|c|}{ Before } & \multicolumn{2}{|l|}{ After } & \multicolumn{2}{|c|}{ Before } & \multicolumn{2}{|l|}{ After } \\
\hline & M & SD & M & SD & M & SD & M & SD \\
\hline WHO hygiene & 0.70 & 0.17 & 0.95 & 0.06 & 0.83 & 0.13 & 0.99 & 0.03 \\
\hline Attitude & 33.40 & 15.07 & 47.60 & 4.07 & 38.97 & 13.48 & 49.00 & 0.00 \\
\hline Beliefs & 36.53 & 12.42 & 40.57 & 10.70 & 48.07 & 3.62 & 49.00 & 0.00 \\
\hline
\end{tabular}

Note. WHO = World Health Organization

Table 3: Simple effects for floor (control-intervention) by time

\begin{tabular}{lllll}
\hline & & \multicolumn{2}{c}{$95 \%$ confidence interval } \\
Time & Mean difference & $\mathrm{p}$ & Lower limit & Upper limit \\
\hline Baseline & 65.50 & 0.175 & -31.05 & 162.05 \\
Postintervention & -74.86 & 0.033 & -143.14 & -6.58 \\
\hline
\end{tabular}


Table 4: Simple effects for time (posttreatment-baseline) by floor

\begin{tabular}{lllll}
\hline & & \multicolumn{2}{c}{$95 \%$ confidence interval } \\
Floor & Mean difference & $\mathrm{p}$ & Lower limit & Upper limit \\
\hline Control & 43.83 & 0.229 & -29.38 & 117.05 \\
Intervention & 184.20 & $<0.001$ & 133.81 & 234.59 \\
\hline
\end{tabular}

The Hand Sanitizer measurement resulted in a significant time by floors interaction $\left(F_{1,26}=10.54, p=\right.$ $\left.0.003,{ }_{p} \eta^{2}=0.288\right)$ The analysis of simple main effects found the following as shown in Table 2. Such that there was no significant difference in hand sanitizer use between control and intervention floors at baseline ( $p=$ $0.175)$. After the intervention training the intervention floors had significantly more hand sanitizer use than the control floors $(p=0.033)$.

The simple main effects contrasting the use of hand sanitizer within the control group and within the treatment group. The control floor did not have a significant change in the use of sanitizer $(p=0.229)$ and the intervention floors showed a significant $(p<0.001)$ use of sanitizer after the intervention (Table 3 ).

The treatment groups showed improved use of sanitizer as depicted in Table 4.

\section{Summary}

The research question guiding this study was as follows: Is there a significant improvement in the handhygiene knowledge, beliefs, attitudes and compliance of CNAs after they undergo the "My Five Moments for Hand Hygiene" intervention? To address this research questions, six hypotheses were developed relating to the hand hygiene knowledge, belief, attitude and compliance of $45 \mathrm{CNAs}$ at a health care facility. An analysis of the data indicated that the intervention using the "My Five Moments for Hand Hygiene" created a significant difference in hand washing knowledge and attitudes and beliefs when compared to pre-intervention scores. However, there was not a significant difference in selfassessed hand washing belief and attitudes.

\section{Discussion of the Results}

The current study was developed as a result of a thorough review and analysis of current and seminal research related to hand hygiene in health care facilities. Consequently, the results of this study must be interpreted as they relate to the body of existing literature. First, the problem of hand hygiene and the use of hand washing as a remedy of that problem are wellsupported in literature. Research literature review put forth an in-depth discussion of hand hygiene knowledge, beliefs and attitudes, as well as the results of previous studies related to the "My Five Moments for Hand Hygiene" approach. The review also explores the use of proxies in other research as "research surrogates."
Hand hygiene compliance is identified in this review as a way of curbing the threat of HAIs. However, there are several constructs that should be defined in order to understand how hand hygiene compliance can be achieved. As such, knowledge, beliefs and attitudes are key factors that were examined and said to have had a significant impact on hand hygiene compliance. To examine knowledge of hand hygiene is to understand what is important to know, why it is important to know and how knowing about hand hygiene can determine when it should be applied for maximum effect. Beliefs about hand hygiene is also critical and has to be examined to determine how negative and wrong beliefs affect hand hygiene compliance. Attitude toward hand hygiene is also a very significant influence on hand hygiene compliance.

According to Gould et al. (2017), hand washing remains the most commonly proposed, cost-effective and reliable way of reducing the risk of cross infection. The review in this study has documented the effectiveness of the "My Five Moments to Hand Hygiene" approach as a best-practice training for hand hygiene compliance. It has led to immediate post-intervention improvement in hand hygiene compliance. This research suggested that hand hygiene remains the most commonly proposed, cost-effective and reliable way of reducing the risk of cross infection. Thus, the results of this research study supported previous research. In examining the results as presented in Tables the intervention group scored higher than the control group at baseline and improved their hygiene scores with the intervention. On the other hand, the control group while having lower hygiene scores had a larger increase in hygiene scores in the post treatment evaluation.

Other researchers have examined the efficacy of implementing strategic approaches to hand hygiene in order to improve hand hygiene in health care facilities. For example, Gould et al. (2017) examined the effectiveness of approaches used to increase hand hygiene during patient care using 14 randomized trials, two non-randomized trials and 10 ITS studies. Therefore, it was appropriate to propose a systematic hand washing technique as an intervention for addressing poor hand hygiene in the health care facility.

\section{Conclusion}

This research project was an attempt to improve hand hygiene practice and compliance among Certified 
Nursing Assistants (CNA) in a health care facility. The project was developed in response to the identified problem that HAI rates need to be reduced in health care facilities. The study was designed as a quasiexperimental research project using a quantitative pretest and posttest design with the evidence-based "My Five Moments for Hand Hygiene" training used as in intervention to improve hand hygiene compliance at a long-term care facility. The "My Five Moments for Hand Hygiene" has been demonstrated through the literature to be an effective intervention for improving hand hygiene among health care staff. The Theory of Planned Behavior was utilized as a framework for the design of the study and the interpretation of study results. TPB has been demonstrated in literature as an effective lens for understanding why health care staff has inadequate hand hygiene practices and how to address the problem. This study was significant because it attempted to provide CNAs in the intervention group with a best-practice training to facilitate new knowledge and create a personal system and a collaborative peer-to-peer initiative to improve hand hygiene compliance as a sustainable approach compared to the applicator technology already in place at this facility. The data for this study was collected using wall hand-sanitizer usage, the WHO Hand Hygiene Knowledge Questionnaire for Healthcare Workers and the Hand Washing Self-Assessment Inventory Scale Questionnaire. These data were analyzed using 2 by 2 factorial ANOVA to determine if there were significant differences in the intervention and control groups pre- and post-intervention. It was determined that the implementation of the "My Five Moments for Hand Hygiene" was effective in increasing the rates of hand hygiene compliance and hand hygiene knowledge. There was not a significant difference between the two participant groups in regard to Hand Washing self-assessment belief and attitude, but both groups demonstrated significant improvement pre- and post-intervention. It can be concluded that this intervention is effective for the improvement of hand hygiene compliance among CNAs at the selected health care facility. The success of this intervention has practical implications for the field as a whole and contributes to the body of literature in this area.

\section{Acknowledgement}

CE Turkett Consultants, LLC Partners, SALT Rehabilitation Clinic-Liberia. My Family and Academic Committee Members and Research Site.

\section{Ethics}

Belmont Report 1978 (Respect for Persons, Beneficence and Justice).

\section{References}

Ajzen, I. and M. Fishbein, 1980. Understanding attitudes and predicting social behavior. J. Personality Soc. Psychol., 62: 98-109. DOI: 10.1037/0022-3514.62.1.98

Ajzen, I., 1985. From Intentions to Actions: A Theory of Planned Behavior. In: Action Control, Kuhl, J. and J. Beckmann (Eds.), SSSP Springer Series in Social Psychology, Springer, Berlin, ISBN-13: 978-3-642-69746-3, pp: 11-39.

Al-Khawaldeh, O.A., M. Al-Hussami and M. Darawad, 2015. Influence of nursing students hand washing knowledge, belief and attitude. J. Health, 7: 572-579. DOI: $10.4236 /$ HEALTH.2015.75068

Boev, C. and Y. Xia, 2015. Nurse-physician collaboration and hospital-acquired infections in critical care. Critical Care Nurse, 35: 66-72. DOI: $10.4037 / \mathrm{CCN} 2015809$

CDCP, 2016. HAI data and statistics. Healthcareassociated infections.

CHC, 2010. Changing hospital culture. Industrial Eng., 42: 14-15.

Chen, J.C., K.S. Wu, S.S.J. Lee, H.S. Lin and H.C. Tsai et al., 2016. Impact of implementation of the world health organization multimodal hand hygiene improvement strategy in a teaching hospital in Taiwan. Am. J. Infect. Control, 44: 222-227. DOI: 10.1016/J.AJIC.2015.10.004

Farhoudi, F., A.S. Dashti, N.G. Davani, G. Sajadi and R. Taghizadeh, 2016. Impact of WHO hand hygiene improvement program implementation: A quasiexperimental trial. BioMed Res. Int.

Faul, F., E. Erdfelder and A. Lang, 2009. G* Power 3: A flexible statistical power analysis program for the social, behavioral and biomedical sciences. Behav. Res. Meth., 39: 175-191. DOI: 10.3758/Bf03193146

Fox, C., T. Wavra, D.A. Drake, D. Mulligan and Y.P. Bennett et al., 2015. Use of a patient hand hygiene protocol to reduce hospital-acquired infections and improve nurses' hand washing. Am. J. Critical Care, 24: 216-224. DOI: 10.4037/AJCC2015898

Gould, D.J., D. Moralejo, N. Drey, J.H. Chudleigh and M. Taljaard, 2017. Interventions to improve hand hygiene compliance in patient care. J. Infect. Prevent., 19: 108-113. DOI: 10.1002/14651858.CD005186.PUB4

HAI, 2014. Healthcare-acquired infections: Culture over campaigns. Health Leaders, 17: 44-48.

Melo de Souza, L., M.F. Ramos, E.S.S. Becker, L.C. Meirelles and S.A.O. Monteiro, 2015. Adherence to the five moments for hand hygiene among intensive care professionals. Rivista Gaucha de Enfermagem.

Moghnieh, R., R. Soboh, D. Abdallah, M. El-Helou and S. Al Hassan et al., 2017. Health care workers' compliance to my 5 moments for hand hygiene: Comparison of 2 interventional methods. Am. J. Infect. Control, 45: 89-91. DOI: 10.1016/J.AJIC.2016.08.012 
Monistrol, O., E. Calbo, M. Riera, C. Nicolas and R. Font et al., 2012. Impact of a hand hygigne educational programme on hospital-acquired infections in medical wards. Clin. Microbiol. Infect., 18: 1212-1218. DOI: 10.1111/J.1469-0691.2011.03735.X

Nabavi, M., M. Alavi-Moghaddam, L. Gachkar and M. Moeinaian, 2013. Knowledge, attitudes and practices study of hand hygiene among, Imam Hossein Hospital's Residents. Iran Red Crescent Med. J., 17: 1-8. DOI: 10.5812/ircmj. 19606

NCPHSBBR, 1978. The Belmont report: Ethical principle and guideline for the protection of human subjects of research.

NHHV, 2018. Kaiser Health News.

O' Boyle, C.A., S.J. Henly and E. Larson, 2001. Understanding adherence to hand hygiene recommendation: Theory of planned behavior. Am. J. Infect. Control.

Pulliam, J.R., 2015. Lower infection rates after introduction of a photocatalytic surface coating. Am. J. Infect. Control, 43: 180-181.

DOI: 10.1016/j.ajic.2014.10.023
Sax, H., B. Allegranzi, I. Uckay, E. Larson and J. Boyce et al., 2007. My five moments for hand hygiene: A user-centered design approach to understand, train, monitor and report hand hygiene. J. Hospital Infect., 67: 9-21. DOI: 10.1016/J.JHIN.2007.06.004

Shinde, M.B. and J.R. Mohite, 2014. A study to assess knowledge, attitude and practices of 5 moments of hand hygiene among nursing staff and students at a tertiary care hospital at Karad. Int. J. Sci. Res., 3: 311-321.

Shobowale, E.O., B. Adegunle and K. Onyedibe, 2016. An assessment of hand hygiene practice of healthcare workers of a semi-urban hospital using the 5 moments of hand hygiene. Nigeria Med. J., 57: 150-154. DOI: 10.4103/0300-1652.184058

WHO, 2017. About save lives: Clean your hands. Clean care is safer care. 